\title{
A new approach to the realization problem for fractional discrete-time linear systems
}

\author{
T. KACZOREK* \\ Faculty of Electrical Engineering, Białystok University of Technology, 45D Wiejska St., 15-351 Bialystok, Poland
}

\begin{abstract}
A new approach to the realization problem for fractional discrete-time linear systems is proposed. A procedure for computation of fractional realizations of given transfer matrices is presented and illustrated by numerical examples.
\end{abstract}

Key words: fractional, linear, discrete-time, system, computation procedure, realization, transfer matrix.

\section{Introduction}

Determination of the state space equations for given transfer matrices is a classical problem, called the realization problem, which has been addressed in many papers and books [1-8]. An overview of the positive realization problem is given in [1, $2,6,9]$. The realization problem for positive continuous-time and discrete-time linear system has been considered in $[6,7$, 10-22] and for linear systems with delays in [6, 10, 15, 2124]. The realization problem for fractional linear systems has been analyzed in $[6,7,25-30]$ for positive $2 \mathrm{D}$ hybrid linear systems in $[24,31,32]$ and for fractional systems with delays in $[33,34]$. A new modified state variable diagram method for determination of positive realizations with reduced number of delays for given proper transfer matrices has been proposed in [35].

In this paper a new approach to the realization problem for fractional discrete-time linear systems will be proposed. The paper is organized as follows. Some preliminaries and problem formulation are given in Sec. 2. In Sec. 3 the solution to the realization problem for fractional discrete-time linear systems is presented and illustrated by numerical examples. Concluding remarks are given in Sec. 4.

The following notation will be used: $\Re$ - the set of real numbers, $\Re^{n \times m}$ - the set of $n \times m$ real matrices, $\Re^{n \times m}(w)$ the set of $n \times m$ rational matrices in $w$ with real coefficients, $Z_{+}$- the set of nonnegative integers, $I_{n}-$ the $n \times n$ identity matrix.

\section{Preliminaries and problem formulation}

Consider the fractional discrete-time linear system

$$
\begin{gathered}
\Delta^{\alpha} x_{i}=A x_{i}+B u_{i}, \quad i \in Z_{+}=\{0,1, \ldots\}, \\
y_{i}=C x_{i}+D u_{i},
\end{gathered}
$$

where

$$
\begin{gathered}
\Delta^{\alpha} x_{i}=\sum_{j=0}^{i} c_{j} x_{i-j}, \\
c_{j}=(-1)^{j}\left(\begin{array}{c}
\alpha \\
j
\end{array}\right) \\
=\left\{\begin{array}{ccc}
1 & \text { for } & j=0 \\
(-1)^{j} \frac{\alpha(\alpha-1) \ldots(\alpha-j+1)}{j !} & \text { for } & j=1,2, \ldots
\end{array},\right.
\end{gathered}
$$

$x_{i} \in \Re^{n}, u_{i} \in \Re^{m}, y_{i} \in \Re^{p}$ are the state, input and output vectors and $A \in \Re^{n \times n}, B \in \Re^{n \times m}, C \in \Re^{p \times n}, D \in \Re^{p \times m}$.

Using the Z-transformation to (1a) and (1b) for zero initial conditions we obtain [6]

$$
\begin{gathered}
Z\left[\Delta^{\alpha} x_{i}\right]=w X(z)=A X(z)+B U(z), \\
i \in Z_{+}=\{0,1, \ldots\} \\
Y(z)=C X(z)+D U(z),
\end{gathered}
$$

where

$$
\begin{gathered}
Z\left[\Delta^{\alpha} x_{i}\right]=\left(1-z^{-1}\right)^{\alpha} X(z)=w(z) X(z)=w X(z), \\
w=w(z)=\left(1-z^{-1}\right)^{\alpha}=\sum_{i=0}^{\infty} c_{i} z^{-i}, \\
X(z)=Z\left[x_{i}\right]=\sum_{i=0}^{\infty} x_{i} z^{-i}, \\
U(z)=Z\left[u_{i}\right], \quad Y(z)=Z\left[y_{i}\right] .
\end{gathered}
$$

From (2) we have the transfer matrix

$$
T(w)=C\left[I_{n} w-A\right]^{-1} B+D .
$$

The transfer matrix $T(z)$ is called proper if and only if

$$
\lim _{w \rightarrow \infty} T(w)=D \in \Re^{p \times m}
$$

and it is called strictly proper if and only if $D=0$.

From (3) we have

$$
\lim _{w \rightarrow \infty} T(w)=D
$$

since $\lim _{w \rightarrow \infty}\left[I_{n} w-A\right]^{-1}=0$.

\footnotetext{
*e-mail: kaczorek@isep.pw.edu.pl
} 
T. Kaczorek

Definition 1. The matrices $A, B, C, D$ are called a fractional realization of a given transfer matrix $T(w)$ if they satisfy the equality (3).

A fractional realization $A, B, C, D$ is called minimal if the dimension of the matrix $A$ is minimal among all realizations of $T(w)$.

The fractional realization problem can be stated as follows. Given a proper transfer matrix $T(w) \in \Re^{p \times m}(w)$ find a fractional realization $A, B, C, D$ of the matrix $T(w)$.

\section{Problem solution}

3.1. Single-input single-output systems. First the essence of the proposed method is presented for single-input singleoutput (SISO) fractional discrete-time linear systems with the transfer function

$$
T(w)=\frac{b_{n} w^{n}+b_{n-1} w^{n-1}+\ldots+b_{1} w+b_{0}}{w^{n}+a_{n-1} w^{n-1}+\ldots+a_{1} w+a_{0}} .
$$

Using (4) for (6) we obtain

$$
D=\lim _{w \rightarrow \infty} T(w)=b_{n}
$$

and

$$
T_{s p}(w)=T(w)-D=\frac{\bar{b}_{n-1} w^{n-1}+\ldots+\bar{b}_{1} w+\bar{b}_{0}}{w^{n}+a_{n-1} w^{n-1}+\ldots+a_{1} w+a_{0}},
$$

where

$$
\bar{b}_{k}=b_{k}-a_{k} b_{n}, \quad k=0,1, \ldots, n-1 .
$$

Therefore, the realization problem has been reduced to finding matrices $A \in \Re^{n \times n}, B \in \Re^{n \times m}, C \in \Re^{p \times n}$ for given strictly proper transfer function (8a).

Multiplying the numerator and the denominator of (8a) by $w^{-n}$ we obtain

$$
T_{s p}(w)=\frac{Y}{U}=\frac{\bar{b}_{n-1} w^{-1}+\ldots+\bar{b}_{1} w^{1-n}+\bar{b}_{0} w^{-n}}{1+a_{n-1} w^{-1}+\ldots+a_{1} w^{1-n}+a_{0} w^{-n}},
$$

where $Y$ and $U$ are the Z-transforms of $y_{i}$ and $u_{i}$, respectively.

Define

$$
E=\frac{U}{1+a_{n-1} w^{-1}+\ldots+a_{1} w^{1-n}+a_{0} w^{-n}} .
$$

From (9) and (10) we have

$$
\begin{gathered}
E=U-\left(a_{n-1} w^{-1}+\ldots+a_{1} w^{1-n}+a_{0} w^{-n}\right) E \\
Y=\left(\bar{b}_{n-1} w^{-1}+\ldots+\bar{b}_{1} w^{1-n}+\bar{b}_{0} w^{-n}\right) E
\end{gathered}
$$

From (11) follows the block diagram shown in Fig. 1.

Assuming as the state variables $x_{1, i}, x_{2, i}, \ldots, x_{n, i}$ the outputs of the delay elements we may write the equations

$$
\begin{gathered}
\Delta^{\alpha} x_{1, i}=x_{2, i}, \\
\Delta^{\alpha} x_{2, i}=x_{3, i}, \\
\vdots \\
\Delta^{\alpha} x_{n-1, i}=x_{n, i}, \\
\Delta^{\alpha} x_{n, i}=-a_{0} x_{1, i}-a_{1} x_{2, i}-\ldots-a_{n-1} x_{n, i}+u_{i}
\end{gathered}
$$

and

$$
y_{i}=\bar{b}_{0} x_{1, i}+\bar{b}_{1} x_{2, i}+\ldots+\bar{b}_{n-1} x_{n, i} .
$$

The Eq. (12) can be written in the form

$$
\begin{gathered}
\Delta^{\alpha} x_{i}=A x_{i}+B u_{i}, \\
y_{i}=C x_{i},
\end{gathered}
$$

where

$$
\begin{gathered}
x_{i}=\left[\begin{array}{ccccc}
x_{1, i} & x_{2, i} & \ldots & x_{n, i}
\end{array}\right]^{T}, \quad i \in Z_{+}, \\
A=\left[\begin{array}{ccccc}
0 & 1 & 0 & \ldots & 0 \\
0 & 0 & 1 & \ldots & 0 \\
\vdots & \vdots & \vdots & \ddots & \vdots \\
0 & 0 & 0 & \ldots & 1 \\
-a_{0} & -a_{1} & -a_{2} & \ldots & -a_{n-1}
\end{array}\right],
\end{gathered}
$$

$$
B=\left[\begin{array}{c}
0 \\
\vdots \\
0 \\
1
\end{array}\right], \quad C=\left[\begin{array}{lllll}
\bar{b}_{0} & \bar{b}_{1} & \ldots & \bar{b}_{n-1}
\end{array}\right] \text {. }
$$

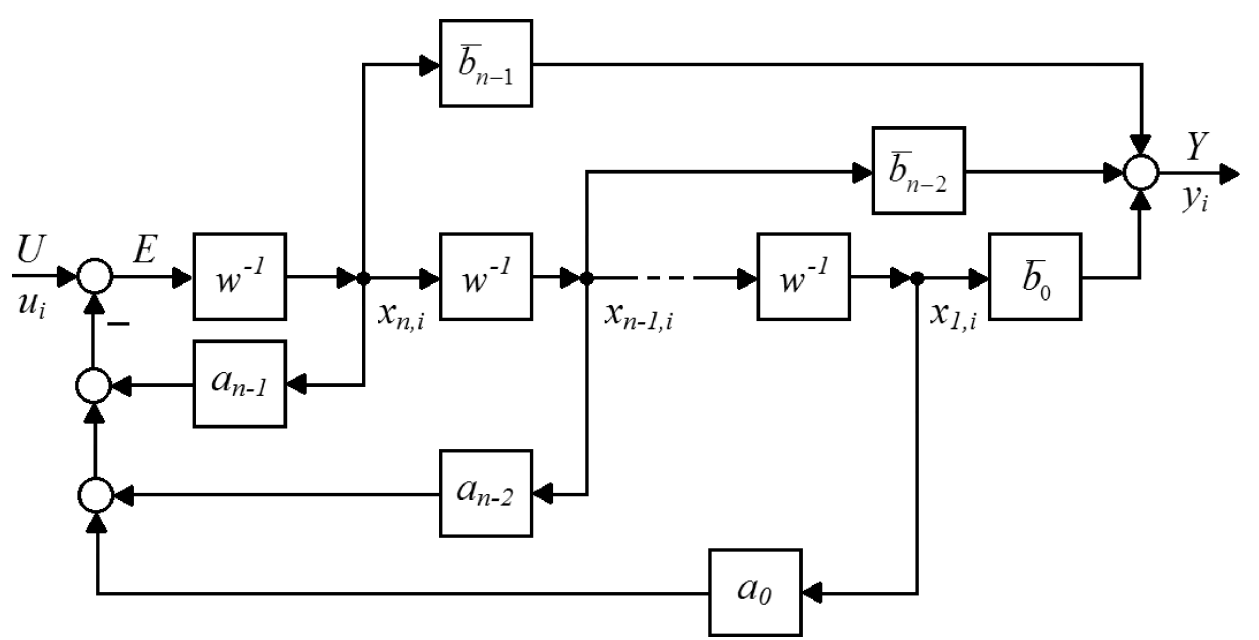

Fig. 1. State diagram for transfer function (9) 
Remark 1. If we choose the state variables so that $x_{k}=$ $x_{n-k+1}$ for $k=1, \ldots, n$ then the realization of (8) has the form

$$
\begin{gathered}
A_{1}=\left[\begin{array}{ccccc}
-a_{n-1} & -a_{n-2} & \cdots & -a_{1} & -a_{0} \\
1 & 0 & \cdots & 0 & 0 \\
\vdots & \vdots & \ddots & \vdots & \vdots \\
0 & 1 & \cdots & 0 & 0 \\
0 & 0 & \cdots & 1 & 0
\end{array}\right], \\
B_{1}=\left[\begin{array}{c}
1 \\
0 \\
\vdots \\
0
\end{array}\right], \\
C_{1}=\left[\begin{array}{lllll}
\bar{b}_{n-1} & \bar{b}_{n-2} & \cdots & \bar{b}_{0}
\end{array}\right] .
\end{gathered}
$$

Remark 2. Note that the transposition (denoted by $T$ ) of the transfer function does change it, i.e. $\left[T_{s p}(w)\right]^{T}=T_{s p}(w)=$ $\left[C\left[I_{n} w-A\right]^{-1} B\right]^{T}=B^{T}\left[I_{n} w-A^{T}\right]^{-1} C^{T}$ and the matrices

$$
\begin{gathered}
A_{2}=A^{T}=\left[\begin{array}{ccccc}
0 & 0 & \cdots & 0 & -a_{0} \\
1 & 0 & \cdots & 0 & -a_{1} \\
0 & 1 & \cdots & 0 & -a_{2} \\
\vdots & \vdots & \ddots & \vdots & \vdots \\
0 & 0 & \cdots & 1 & -a_{n-1}
\end{array}\right], \\
B_{2}=C^{T}=\left[\begin{array}{cc}
\bar{b}_{0} \\
\bar{b}_{1} \\
\vdots \\
\bar{b}_{n-1}
\end{array}\right], \\
C_{2}=B^{T}=\left[\begin{array}{llll}
0 & \cdots & 0 & 1
\end{array}\right]
\end{gathered}
$$

and

$$
\begin{gathered}
A_{3}=A_{1}^{T}=\left[\begin{array}{ccccc}
-a_{n-1} & 1 & 0 & \cdots & 0 \\
-a_{n-2} & 0 & 1 & \ldots & 0 \\
\vdots & \vdots & \vdots & \ddots & \vdots \\
-a_{1} & 0 & 0 & \ldots & 1 \\
-a_{0} & 0 & 0 & \cdots & 0
\end{array}\right], \\
B_{3}=C_{1}^{T}=\left[\begin{array}{c}
\bar{b}_{n-1} \\
\bar{b}_{n-2} \\
\vdots \\
\bar{b}_{0}
\end{array}\right], \\
C_{3}=B_{1}^{T}=\left[\begin{array}{cccc}
1 & 0 & \cdots & 0
\end{array}\right]
\end{gathered}
$$

are also the realizations of the transfer function (8).

Example 1. Find the fractional realization of the transfer function

$$
T(w)=\frac{2 w^{2}+11 w+10}{w^{2}+3 w+4} .
$$

Using (7) we obtain

$$
D=\lim _{w \rightarrow \infty} T(w)=2
$$

and

$T_{s p}(w)=T(w)-D=\frac{5 w+2}{w^{2}+3 w+4}=\frac{5 w^{-1}+2 w^{-2}}{1+3 w^{-1}+4 w^{-2}}$.

In this case we have

$$
E=\frac{U}{1+3 w^{-1}+4 w^{-2}}
$$

and

$$
\begin{gathered}
E=U-\left(3 w^{-1}+4 w^{-2}\right) E, \\
Y=\left(5 w^{-1}+2 w^{-2}\right) E .
\end{gathered}
$$

The block diagram corresponding to (22) is shown in Fig. 2.

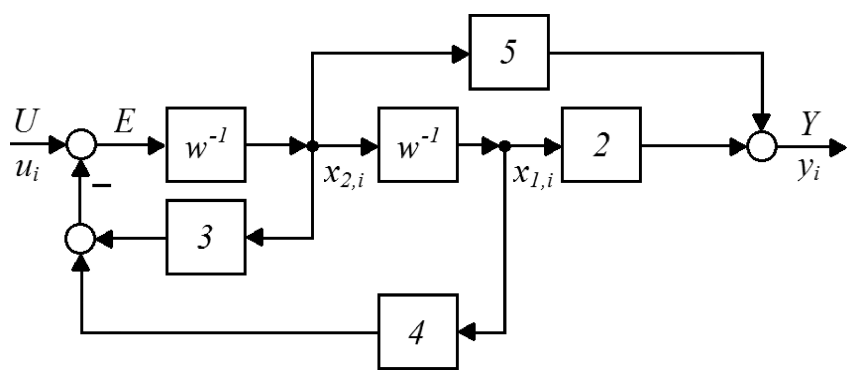

Fig. 2. State diagram for transfer function (20)

For the choice of the state variables shown in Fig. 2 we obtain the equations

$$
\begin{gathered}
\Delta^{\alpha} x_{1, i}=x_{2, i}, \\
\Delta^{\alpha} x_{2, i}=-4 x_{1, i}-3 x_{2, i}+u, \\
y_{i}=2 x_{1, i}+5 x_{2, i}
\end{gathered}
$$

and the realization

$$
A=\left[\begin{array}{cc}
0 & 1 \\
-4 & -3
\end{array}\right], \quad B=\left[\begin{array}{l}
0 \\
1
\end{array}\right], \quad C=\left[\begin{array}{ll}
2 & 5
\end{array}\right] \text {. }
$$

3.2. Multi-input multi-output systems. Consider a strictly proper transfer matrix $T_{s p}(w) \in \Re^{p \times m}(w)$. Let

$$
\begin{gathered}
D_{i}(w)=w^{d_{i}}-\left(a_{i, d_{i}-1} w^{d_{i}-1}+\ldots+a_{i, 1} w+a_{i, 0}\right), \\
i=1, \ldots, m
\end{gathered}
$$

be the least common denominator of all entries of the $i$-th column of $T_{s p}(w)$.

Using (25) we may write $T_{s p}(w)$ in the form

$$
T_{s p}(w)=\left[\begin{array}{ccc}
\frac{N_{11}(w)}{D_{1}(w)} & \cdots & \frac{N_{1 m}(w)}{D_{m}(w)} \\
\vdots & \ddots & \vdots \\
\frac{N_{p 1}(w)}{D_{1}(w)} & \cdots & \frac{N_{p m}(w)}{D_{m}(w)}
\end{array}\right]=N(w) D^{-1}(w)
$$

where

$$
\begin{aligned}
& N(w)=\left[\begin{array}{ccc}
N_{11}(w) & \cdots & N_{1 m}(w) \\
\vdots & \ddots & \vdots \\
N_{p 1}(w) & \cdots & N_{p m}(w)
\end{array}\right] \\
& D(w)=\operatorname{diag}\left[\begin{array}{lll}
D_{1}(w) & \cdots & D_{m}(w)
\end{array}\right] \text {. }
\end{aligned}
$$


From (25) it follows that

$$
D(w)=\operatorname{diag}\left[\begin{array}{lll}
w^{d_{i}} & \cdots & w^{d_{m}}
\end{array}\right]-\bar{A}_{m} W,
$$

where

$$
\begin{gathered}
\bar{A}_{m}=\operatorname{blockdiag}\left[\begin{array}{ccc}
a_{1} & \cdots & a_{m}
\end{array}\right], \\
a_{i}=\left[\begin{array}{llll}
a_{i, 0} & \cdots & a_{i, d_{i}-1}
\end{array}\right], \\
W=\operatorname{blockdiag}\left[\begin{array}{lll}
W_{1} & \cdots & W_{m}
\end{array}\right], \\
W_{i}=\left[\begin{array}{llll}
1 & w & \cdots & w^{d_{i}-1}
\end{array}\right] .
\end{gathered}
$$

Note that if

$$
N_{i j}(w)=c_{i j}^{d_{j}-1} w^{d_{j}-1}+\ldots+c_{i j}^{1} w+c_{i j}^{0},
$$

then

$$
N(w)=C W,
$$

where

$$
C=\left[\begin{array}{ccccccccc}
c_{11}^{0} & c_{11}^{1} & \cdots & c_{11}^{d_{1}-1} & \cdots & c_{1 m}^{0} & c_{1 m}^{1} & \cdots & c_{1 m}^{d_{m}-1} \\
\vdots & \vdots & \vdots & \vdots & \ddots & \vdots & \vdots & \vdots & \vdots \\
c_{p 1}^{0} & c_{p 1}^{1} & \cdots & c_{p 1}^{d_{1}-1} & \cdots & c_{p m}^{0} & c_{p m}^{1} & \cdots & c_{p m}^{d_{m}-1}
\end{array}\right] .
$$

We shall show that the matrices

$$
\begin{aligned}
& A=\operatorname{blockdiag}\left[\begin{array}{lll}
A_{1} & \cdots & A_{m}
\end{array}\right], \\
& A_{i}=\left[\begin{array}{ccccc}
0 & 1 & 0 & \cdots & 0 \\
0 & 0 & 1 & \cdots & 0 \\
\vdots & \vdots & \vdots & \ddots & \vdots \\
0 & 0 & 0 & \cdots & 1 \\
a_{i, 0} & a_{i, 1} & a_{i, 2} & \cdots & a_{i, d_{i}-1}
\end{array}\right] \text {, } \\
& i=1, \ldots, m \text {, } \\
& B=\operatorname{blockdiag}\left[\begin{array}{lll}
b_{1} & \cdots & b_{m}
\end{array}\right], \\
& b_{i}=\left[\begin{array}{llll}
0 & \cdots & 0 & 1
\end{array}\right]^{T} \in \Re^{d_{i}}, \quad i=1, \ldots, m
\end{aligned}
$$

and (28c) are the desired realization of (26).

Using (27) and (28) it is easy to verify that

$$
b_{i} D_{i}(w)=\left[I_{n} w-A_{i}\right]\left[\begin{array}{c}
1 \\
w \\
\vdots \\
w^{d_{i}-1}
\end{array}\right]
$$

and

$$
B D(w)=\left[I_{n} w-A\right] W .
$$

Premultiplication of (31) by $C\left[I_{n} w-A\right]^{-1}$ and postmultiplication by $D^{-1}(w)$ yields

$$
\begin{gathered}
C\left[I_{n} w-A\right]^{-1} B=C W D^{-1}(w) \\
=N(w) D^{-1}(w)=T_{s p}(w) .
\end{gathered}
$$

Therefore, we have the following procedure for finding a fractional realization of a given proper transfer matrix $T(w)$.

\section{Procedure 1.}

Step 1. Using (4) find the matrix $D$ and the strictly proper transfer matrix $T_{s p}(w)$.
Step 2. Find the least common denominators $D_{1}(w), \ldots$, $D_{m}(w)$ and write $T_{s p}(w)$ in the form (26).

Step 3. Knowing $D(w)$ find the indices $d_{1}, \ldots, d_{m}$ and the matrices $W$ and $\bar{A}_{m}$.

Step 4. Knowing $N(w)$ find the matrix $C$ defined by (28c).

Step 5. Using (29) find the matrices $A$ and $B$.

Remark 3. Similar results can be obtained for the least common denominator of all entries of the $j$-th row of $T_{s p}(w)$.

Example 2. Find the fractional realization of the transfer matrix

$$
T(w)=\left[\begin{array}{cc}
\frac{2 w+1}{w} & \frac{w+3}{w+1} \\
\frac{3 w+8}{w+2} & \frac{2 w+5}{w+2}
\end{array}\right] .
$$

Using Procedure 1 and (33) we obtain the following:

Step 1. Using (4) and (33) we obtain

$$
\begin{gathered}
D=\lim _{w \rightarrow \infty} T(w) \\
=\lim _{w \rightarrow \infty}\left[\begin{array}{cc}
\frac{2 w+1}{w} & \frac{w+3}{w+1} \\
\frac{3 w+8}{w+2} & \frac{2 w+5}{w+2}
\end{array}\right]=\left[\begin{array}{ll}
2 & 1 \\
3 & 2
\end{array}\right]
\end{gathered}
$$

and

$$
T_{s p}(w)=T(w)-D=\left[\begin{array}{cc}
\frac{1}{w} & \frac{2}{w+1} \\
\frac{2}{w+2} & \frac{1}{w+2}
\end{array}\right] .
$$

Step 2. From (35) we have $D_{1}(w)=w(w+2), D_{2}(w)=$ $(w+1)(w+2)$ and

$$
T_{s p}(w)=N(w) D^{-1}(w),
$$

where

$$
\begin{gathered}
N(w)=\left[\begin{array}{cc}
w+2 & 2(w+2) \\
2 w & w+1
\end{array}\right], \\
D(w)=\left[\begin{array}{cc}
w(w+2) & 0 \\
0 & (w+1)(w+2)
\end{array}\right] .
\end{gathered}
$$

Step 3. From (36b) we have $d_{1}=d_{2}=2$ and

$$
W=\left[\begin{array}{cc}
1 & 0 \\
w & 0 \\
0 & 1 \\
0 & w
\end{array}\right], \quad \bar{A}_{m}=\left[\begin{array}{cccc}
0 & -2 & 0 & 0 \\
0 & 0 & -2 & -3
\end{array}\right]
$$

since

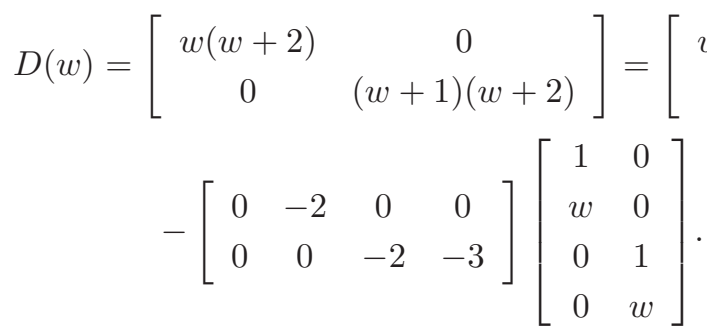


Step 4. Using (36b) we obtain

$$
\begin{gathered}
N(w)=\left[\begin{array}{cc}
w+2 & 2 w+4 \\
2 w & w+1
\end{array}\right] \\
=\left[\begin{array}{llll}
2 & 1 & 4 & 2 \\
0 & 2 & 1 & 1
\end{array}\right]\left[\begin{array}{cc}
1 & 0 \\
w & 0 \\
0 & 1 \\
0 & w
\end{array}\right]=C W
\end{gathered}
$$

and

$$
C=\left[\begin{array}{llll}
2 & 1 & 4 & 2 \\
0 & 2 & 1 & 1
\end{array}\right] .
$$

Step 5. Using (29) and (37) we obtain

$$
A=\left[\begin{array}{cccc}
0 & 1 & 0 & 0 \\
0 & -2 & 0 & 0 \\
0 & 0 & 0 & 1 \\
0 & 0 & -2 & -3
\end{array}\right], \quad B=\left[\begin{array}{ll}
0 & 0 \\
1 & 0 \\
0 & 0 \\
0 & 1
\end{array}\right] .
$$

The desired fractional realization of (33) is given by (40), (39b) and (34).

\section{Concluding remarks}

A new approach to finding fractional realizations of given transfer matrices of discrete-time linear systems has been proposed. It has been shown that for any given proper transfer matrix there exist always many fractional realizations. A procedure for computation a fractional realization of a given transfer matrix has been proposed. The effectiveness of the procedure has been demonstrated on numerical examples. The classical Gilbert method [29] can also be applied to compute the fractional realizations of the given transfer matrices of discrete-time linear systems.

The presented method can be easily extended to positive fractional linear discrete-time systems without and with delays.

Acknowledgements. This work was supported by the grant No. S/WE/1/2015.

\section{REFERENCES}

[1] L. Benvenuti and L. Farina, "A tutorial on the positive realization problem", IEEE Trans. on Automatic Control 49 (5), 651-664 (2004).

[2] L. Farina and S. Rinaldi, Positive Linear Systems. Theory and Applications, J. Wiley, New York, 2000.

[3] T. Kaczorek, "Existence and determination of the set of Metzler matrices for given stable polynomials", Int. J. Appl. Math. Comput. Sci. 22 (2), 389-399 (2012).

[4] T. Kaczorek, Linear Control Systems, vol. 1, Research Studies Press, J. Wiley, New York, 1992.

[5] T. Kaczorek, Polynomial and Rational Matrices, Springer, London, 2009.

[6] T. Kaczorek and Ł. Sajewski, The Realization Problem for Positive and Fractional Systems, Springer, London, 2014.
[7] T. Kaczorek, Selected Problems in Fractional Systems Theory, Springer, London, 2011.

[8] U. Shaker and M. Dixon, "Generalized minimal realization of transfer-function matrices", Int. J. Contr. 25 (5), 785-803 (1977).

[9] T. Kaczorek, Positive $1 D$ and $2 D$ Systems, Springer, London, 2002.

[10] T. Kaczorek, "A realization problem for positive continuoustime linear systems with reduced numbers of delays", Int. J. Appl. Math. Comput. Sci. 16 (3), 325-331 (2006).

[11] T. Kaczorek, "Computation of positive stable realizations for discrete-time linear systems", Computational Problems of Electrical Engineering 2 (1), 41-48 (2012).

[12] T. Kaczorek, "Computation of positive stable realizations for linear continuous-time systems", Bull. Pol. Ac.: Tech. 59 (3), 273-281 (2011).

[13] T. Kaczorek, "Computation of realizations of discrete-time cone systems", Bull. Pol. Ac.: Tech. 54 (3), 347-350 (2006).

[14] T. Kaczorek, "Positive and asymptotically stable realizations for descriptor discrete-time linear systems", Bull. Pol. Ac.: Tech. 61 (1), 229-237 (2013).

[15] T. Kaczorek, "Positive minimal realizations for singular discrete-time systems with delays in state and delays in control”, Bull. Pol. Ac.: Tech. 53 (3), 293-298 (2005).

[16] T. Kaczorek, "Positive realizations for descriptor continuoustime linear systems", Measurement Automation and Monitoring 56 (9), 815-818 (2012).

[17] T. Kaczorek, "Positive realizations for descriptor discrete-time linear systems", Acta Mechanica et Automatica, 6 (2), 58-61 (2012).

[18] T. Kaczorek, "Positive stable realizations of continuous-time linear systems", Proc. Conf. Int. Inf. and Eng. Syst. 1, CDROM (2012).

[19] T. Kaczorek, "Positive stable realizations of discrete-time linear systems”, Bull. Pol. Ac.: Tech. 60 (3), 605-616 (2012).

[20] T. Kaczorek, "Positive stable realizations with system Metzler matrices", Archives of Control Sciences 21 (2), 167-188 (2011).

[21] T. Kaczorek, "Realization problem for positive discretetime systems with delays", System Science 30 (4), 117-130 (2004).

[22] T. Kaczorek, "Realization problem for positive multivariable discrete-time linear systems with delays in the state vector and inputs", Int. J. Appl. Math. Comput. Sci. 16 (2), 101-106 (2006).

[23] T. Kaczorek, "Determination of positive realizations with reduced numbers of delays or without delays for discrete-time linear systems", Archives of Control Sciences 22 (4), 371-384 (2012).

[24] T. Kaczorek, "Positive realizations with reduced numbers of delays for 2-D continuous-discrete linear systems", Bull. Pol. Ac.: Tech. 60 (4), 835-840 (2012).

[25] T. Kaczorek, "Positive stable realizations for fractional descriptor continuous-time linear systems", Archives of Control Sciences 22 (3), 255-265 (2012).

[26] T. Kaczorek, "Positive stable realizations of fractional continuous-time linear systems", Int. J. Appl. Math. Comput. Sci. 21 (4), 697-702 (2011).

[27] T. Kaczorek, "Realization problem for descriptor positive fractional continuous-time linear systems", Theory and Applications of Non-integer Order Systems, eds. W. Mitkowski, pp. 3-13, Springer, London, 2013. 
[28] T. Kaczorek, "Realization problem for fractional continuoustime systems", Archives of Control Sciences 18 (1), 43-58 (2008).

[29] Ł. Sajewski, "Positive stable minimal realization of fractional discrete-time linear systems", Advances in the Theory and Applications of Non-integer Order Systems eds. W. Mitkowski, pp. 257, 15-30, Springer, London, 2013.

[30] Ł. Sajewski, "Positive stable realization of fractional discretetime linear systems", Asian J. Control 16 (3), DOI: 10.1002/asjc.750 (2014).

[31] T. Kaczorek, "Positive realizations of hybrid linear systems described by the general model using state variable diagram method", J. Automation, Mobile Robotics and Intelligent Systems 4, 3-10 (2010).
[32] T. Kaczorek, "Realization problem for positive 2D hybrid systems", COMPEL 27 (3), 613-623 (2008).

[33] Ł. Sajewski, "Positive realization of fractional continuous-time linear systems with delays", Measurement Automation and Monitoring 58 (5), 413-417 (2012).

[34] L. Sajewski, "Positive realization of fractional discrete-time linear systems with delays", Measurements, Automatics, Robotics 2, CD-ROM, (2012).

[35] T. Kaczorek, "A modified state variables diagram method for determination of positive realizations of linear continuoustime systems with delays", Int. J. Appl. Math. Comput. Sci. 22 (4), 897-905 (2012). 